Research Paper

\title{
Association of EGFR mutations and HMGBI genetic polymorphisms in lung adenocarcinoma patients
}

\author{
Yi-Liang Wu1 1,2,3,\#, Ming-Hsien Chien ${ }^{4,5, \#, ~ Y i n g-E r h ~ C h o u ~ 2,6, ~ J e r-H w a ~ C h a n g 5,7,8, ~ T u-C h e n ~ L i u ~}{ }^{1,9}$, Thomas \\ Chang-Yao Tsao ${ }^{2,10}$, Ming-Chih Chou ${ }^{1,3}$, Shun-Fa Yang1,6, 1 \\ 1. Institute of Medicine, Chung Shan Medical University, Taichung, Taiwan \\ 2. School of Medicine, Chung Shan Medical University, Taichung, Taiwan \\ 3. Department of Surgery, Chung Shan Medical University Hospital, Taichung, Taiwan \\ 4. Graduate Institute of Clinical Medicine, College of Medicine, Taipei Medical University, Taipei, Taiwan \\ 5. Pulmonary Research Center, Wan Fang Hospital, Taipei Medical University, Taipei, Taiwan \\ 6. Department of Medical Research, Chung Shan Medical University Hospital, Taichung, Taiwan \\ 7. Division of Pulmonary Medicine, Department of Internal Medicine, School of Medicine, College of Medicine, Taipei Medical University, Taipei, Taiwan \\ 8. Division of Pulmonary Medicine, Department of Internal Medicine, Wan Fang Hospital, Taipei Medical University, Taipei, Taiwan \\ 9. Department of Chest Medicine, Cheng-Ching General Hospital, Taichung, Taiwan \\ 10. Division of Chest, Department of Internal Medicine, Chung Shan Medical University Hospital, Taichung, Taiwan \\ \#These authors contributed equally to this work. \\ $\square$ Corresponding author: Shun-Fa Yang, PhD. Institute of Medicine, Chung Shan Medical University, 110 Chien-Kuo N. Road, Section 1, Taichung 40201, \\ Taiwan. Fax: 886-4-24723229. E-mail: ysf@csmu.edu.tw \\ (c) Ivyspring International Publisher. This is an open access article distributed under the terms of the Creative Commons Attribution (CC BY-NC) license \\ (https://creativecommons.org/licenses/by-nc/4.0/). See http://ivyspring.com/terms for full terms and conditions.
}

Received: 2018.10.31; Accepted: 2019.04.11; Published: 2019.06.02

\begin{abstract}
High-mobility group protein box 1 (HMGBI) is overexpressed and reported to be a prognostic factor in patients with non-small-cell lung cancer (NSCLC). Epidermal growth factor receptor (EGFR) mutants play an important role in NSCLC progression. The aim of this study was to explore potential associations between genetic polymorphisms of HMGBI and EGFR mutations in a cohort that included 280 patients with NSCLC, some of whom were smokers and others who never smoked. Four tagged single-nucleotide polymorphisms (SNPs) of HMGBI were detected by a TaqMan-based real-time polymerase chain reaction (PCR) in patients. We found that after adjusting for other covariates, NSCLC patients who smoked and who respectively had CG, CT, and TC heterozygotes of HMGBI rs2249825, rs 1045411, and rs 1360485, were at lower risk of developing mutant EGFR, compared to those patients with wild-type homozygotes. Moreover, significant inverse associations between the CG and CG + GG genotypes of HMGBI rs2249825 and the EGFR hotspot mutation, an exon 19 in-frame deletion, were also observed among NSCLC patients. Within patients harboring mutant EGFR, HMGBI rs1360485 C (TC + CC) allele carriers were at higher risk of developing poorly differentiated cancer types (odds ratio $=5.493,95 \%$ confidence interval: 1.130 26.696, $p=0.019$ ), compared to patients with TT homozygotes. Furthermore, we found that $H M G B I$ rs 1360485 polymorphisms seemed to be related to susceptibility to developing poorly differentiated cancer linked to tobacco consumption in EGFR mutant patients. In conclusion, our results suggested that $H M G B I$ variants are significantly inversely associated with EGFR mutations among NSCLC patients who smoked. HMGBI variants and tobacco consumption might contribute to the pathological development of NSCLC.
\end{abstract}

Key words: High-mobility group protein box 1, Polymorphism, Susceptibility, Epidermal growth factor receptor, Non-small-cell lung cancer

\section{Introduction}

Lung cancer is one of the most prevalent cancers globally and is by far the leading cause of death among males and females worldwide [1]. Small-cell lung cancer (SCLC) and non-SCLC (NSCLC) are two 
principal groups of lung cancer; the latter type comprises of $85 \% \sim 90 \%$ of lung cancer diagnoses. NSCLC mainly includes the histologic types of adenocarcinoma (ADC) and squamous cell carcinoma (SCC) [2]. During the last decade, there have been major breakthroughs in many aspects of diagnosing and treating NSCLC, including surgery, chemotherapy, radiation therapy, and targeted drug therapy. However, understanding of the mechanisms underlying NSCLC development and progression remains limited, and effective indicators of an early diagnosis of NSCLC are still lacking. Among the many risk factors known to be associated with a higher lung cancer risk, tobacco smoking appears to have the strongest association [3]. Only about $10 \% \sim 15 \%$ of lung cancer cases occur in people who have never smoked, which indicates that other factors such as genetic polymorphisms may play a role in determining disease risk and prognosis in lung cancer [4]. About $8 \%$ of patients have lung cancer solely due to inherited factors, and the contribution of hereditary factors in determining disease risk is also supported by statistics indicating a 2.4-fold increased lung cancer risk in people who are direct relatives of lung cancer patients [5].

The epidermal growth factor receptor (EGFR) is a tyrosine kinase receptor encoded by a gene located on the short arm of chromosome 7 . Autophosphorylation of EGFR tyrosine kinase domains leads to activation of downstream proteins that mediate cell proliferation and cell survival [6] and was reported to play an important role in tumorigenesis and progression of lung adenocarcinomas [7]. The EGFR gene was reported to harbor mutations and/or polymorphisms that increase susceptibility to lung cancer [8]. Most of these mutations/polymorphisms exist within the catalytic kinase domain, which increases EGFR activity. The most common somatic hotspot mutations in the tyrosine kinase domain of the EGFR gene are the in-frame deletion in exon 19 and a substitution mutation of lysine for arginine at amino acid position 858 (L858R) in exon 21. Mutations of L858R and the in-frame deletion in exon 19 were found to be more frequent in adenocarcinoma than other NSCLC and were suggested to promote cell viability [7]. Among adenocarcinoma patients, these EGFR mutations are more common in Asian patients, female patients, and patients who have never smoked [9]. Recently, genetic polymorphisms associated with susceptibility to various somatic mutations were described, including EGFR mutations [10-12], implying that EGFR mutations are linked to an individual's genetic background. In addition, genomic DNA is continuously being damaged by mutagens from external agents, such as tobacco smoke in the environment [13]. In the human body, carcinogen-associated DNA damage must be repaired to maintain correct genetic information. If damaged DNA cannot be repaired, DNA lesions will form, such as EGFR mutations.

High-mobility group box 1 (HMGB1) is a highly conserved nuclear protein widely expressed in mammalian cells, and it plays a critical role in transcriptional regulation and chromatin construction. Loss of HMGB1 can increase DNA damage caused by potent carcinogens in cigarette smoke (such as benzo[a]pyrene diol epoxide) [14] and chemotherapeutic drugs (such as cisplatin) [15]. The binding of HMGB1 to DNA lesions can facilitate DNA repair, the response to DNA damage, and damage-induced chromatin remodeling; thus, it might prevent a carcinogenic or mutagenic outcome after exposure to DNA-damaging agents [16]. In addition to the nuclear function of HMGB1, HMGB1 was reported to be released into the extracellular matrix, where it exerts crucial functions in immunity, inflammation, and carcinogenesis through its diverse receptors such as the receptor for advanced glycation end-products (RAGE), the toll-like receptor (TLR) 2, and TLR 4 [17]. HMGB1 plays a pivotal role in the diagnosis and prognosis of NSCLC, especially with adenocarcinomas [18]. HMGB1 was upregulated in the serum of progressive NSCLC patients and associated with shorter overall survival and disease-free survival times. Overexpression of HMGB1 correlates with the proliferation, metastasis, and chemotherapy resistance of lung adenocarcinomas. HMGB1 may serve as an important risk factor for the development of lung cancer [19].

Investigation of polymorphisms of HMGB1 genes may contribute to our understanding of how cigarette smoke-derived carcinogen metabolism and DNA repair mechanisms affect the development of EGFR mutations in NSCLC. In this study, four polymorphisms of HMGB1 genes, including rs1412125, rs2249825, rs1045411, and rs1360485, were examined to study their associations with susceptibility to EGFR mutations in patients with lung adenocarcinoma.

\section{Material and Methods}

\section{Patient characteristics and consent}

Between 2012 and 2015, 280 lung adenocarcinoma patients with wild-type EGFR (67 men and 44 women; mean age $=65.36 \pm 13.42$ years) or a mutant EGFR (60 men and 109 women; mean age $=65.76 \pm 13.57$ years) were consecutively recruited from Taichung Cheng-Ching General Hospital 
(Taichung, Taiwan). Written ethical consent was obtained from all patients. The study was approved by the Institutional Review Board of Cheng-Ching General Hospital. Data obtained from medical records of each patient included demographics (age and sex), lifestyle variables (tobacco smoking status), and tumor stage and differentiation. Clinical information of patients was staged at the time of diagnosis following the tumor/node/metastasis staging system of the American Joint Committee on Cancer (AJCC).

\section{Selection of single nucleotide polymorphisms (SNPs) of the HMGBI gene}

We genotyped four HMGB1 SNPs including rs1412125 (-1615T/C; promoter region), rs2249825 (3814C/G; intron 1), rs1360485 (T/C; 3' untranslated region (3' UTR)) and rs1045411 (2262C/T; 3' UTR) selected according to Chinese HapMap data. Minor allelic frequencies of these SNPs were all $>5 \%$. These four polymorphisms are well defined and have been widely evaluated the association with a broad range of cancers such as oral squamous cell carcinoma, hepatocellular carcinoma, cervical cancer, colorectal cancer, breast cancer and lung cancer, especially in Han Chinese population [20]. In addition, the minor allele frequencies of these SNPs were all $\geq 5 \%$.

\section{Genomic DNA extraction from blood and HMGBI genotyping}

Genomic DNA was extracted from preserved whole blood in EDTA anti-coagulant tubes using a QIAamp DNA Blood Mini Kit (Qiagen, Valencia, CA, USA) according to the manufacturer's protocol as previously described [21-23]. The TaqMan SNP Genotyping Assay with an ABI StepOnePlus ${ }^{\mathrm{TM}}$ Real-Time PCR System (Applied Biosystems, Foster City, CA, USA) was used to analyze allelic discrimination of HMGB1 SNPs. Allelic frequencies were determined using ABI SDS vers. 3.0 software.

\section{Genomic DNA extraction from tumor tissues and EGFR gene sequencing}

To acquire genomic DNA from tumor-frozen specimens and paraffin-embedded tissues, a QIAamp DNA Tissue kit (Qiagen) was used to extract genomic DNA according to the manufacturer's protocol. DNA sequencing of the EGFR gene in exon 18-21 was amplified by a polymerase chain reaction (PCR), and finally subjected to a DNA sequencing reaction as described previously [9].

\section{Statistical analysis}

The distributions of genotype frequencies and demographic characteristics were compared between EGFR wild-type (WT) and mutation type in lung adenocarcinoma patients by using Mann-Whitney $U$ test. Odds ratio (ORs) and their 95\% confidence intervals (CIs) were assessed using logistic regression models. $p<0.05$ indicated statistically significant differences. Data were analyzed using SAS (version 9.1, 2005; SAS Institute Inc., Cary, NC).

\section{Results}

\section{Demographic and clinical characteristics of patients with lung adenocarcinoma}

Our study recruited 280 lung adenocarcinoma patients who were further divided into EGFR wild-type (WT) $(n=111 ; 39.6 \%)$ and mutation type $(n$ $=169 ; 60.4 \%)$ groups. With regard to the EGFR mutation status, 79 subjects had L858R mutations, 81 had exon19 in-frame deletions, and nine had other mutations. The demographic, lifestyle, and clinical characteristics of both recruited groups with the WT and mutant EGFR are shown in Table 1. Between these two groups, there was no significant difference in the distribution of age, clinical stage, or TNM stage. Compared to the group with the WT EGFR, subjects with the mutant EGFR were significantly observed in females $(64.5 \%$ vs. $39.6 \%)$ and those who had never smoked $(77.5 \%$ vs. $45.0 \%)$, and those who had more well-differentiated $(12.4 \%$ vs. $7.2 \%)$ and moderately differentiated tumors $(81.7 \%$ vs. $72.1 \%)$.

Table 1. Demographics and clinical characteristics of 280 patients with lung adenocarcinoma and their epidermal growth factor mutation status

\begin{tabular}{|c|c|c|c|}
\hline Variable & $\begin{array}{l}\text { Wild type }(\mathrm{N}=111) \\
\mathrm{n}(\%)\end{array}$ & $\begin{array}{l}\text { EGFR mutation } \\
(\mathrm{N}=169) \mathrm{n}(\%)\end{array}$ & $p$ value \\
\hline \multicolumn{4}{|l|}{ Age } \\
\hline Mean \pm SD & $65.36 \pm 13.42$ & $65.76 \pm 13.57$ & $p=0.810$ \\
\hline \multicolumn{4}{|l|}{ Gender } \\
\hline Male & $67(60.4 \%)$ & $60(35.5 \%)$ & $p<0.001$ \\
\hline Female & $44(39.6 \%)$ & $109(64.5 \%)$ & \\
\hline \multicolumn{4}{|c|}{$\begin{array}{l}\text { Cigarette smoking } \\
\text { status }\end{array}$} \\
\hline Never-smoker & $50(45.0 \%)$ & $131(77.5 \%)$ & $p<0.001$ \\
\hline Ever-smoker & $61(55.0 \%)$ & $38(22.5 \%)$ & \\
\hline \multicolumn{4}{|l|}{ Stage } \\
\hline $\mathrm{I}+\mathrm{II}$ & $26(23.4 \%)$ & $47(27.8 \%)$ & $p=0.413$ \\
\hline $\mathrm{III}+\mathrm{IV}$ & $85(76.6 \%)$ & $122(72.2 \%)$ & \\
\hline \multicolumn{4}{|l|}{ Tumor T status } \\
\hline $\mathrm{T} 1+\mathrm{T} 2$ & $60(54.1 \%)$ & $108(63.9 \%)$ & $p=0.100$ \\
\hline $\mathrm{T} 3+\mathrm{T} 4$ & $51(45.9 \%)$ & $61(36.1 \%)$ & \\
\hline \multicolumn{4}{|c|}{ Lymph node status } \\
\hline Negative & $29(26.1 \%)$ & $54(32.0 \%)$ & $p=0.29$ \\
\hline Positive & $82(73.9 \%)$ & $115(68.0 \%)$ & \\
\hline \multicolumn{4}{|c|}{ Distant metastasis } \\
\hline Negative & $54(48.6 \%)$ & $80(47.3 \%)$ & $p=0.830$ \\
\hline Positive & $57(51.4 \%)$ & $89(52.7 \%)$ & \\
\hline \multicolumn{4}{|c|}{ Cell differentiation } \\
\hline Well & $8(7.2 \%)$ & $21(12.4 \%)$ & $p<0.001$ \\
\hline Moderately & $80(72.1 \%)$ & $138(81.7 \%)$ & \\
\hline Poorly & $23(20.7 \%)$ & $10(5.9 \%)$ & \\
\hline
\end{tabular}


Table 2. Distribution frequency of high-mobility group box 1 (HMGBI) genotypes of patients with lung adenocarcinoma and multiple logistic regression analysis of epidermal growth factor receptor mutations associated with the cigarette smoking status.

\begin{tabular}{|c|c|c|c|c|c|c|c|c|c|}
\hline \multicolumn{4}{|c|}{ All cases $(N=280)$} & \multicolumn{3}{|c|}{ Non-smokers $(N=181)$} & \multicolumn{3}{|c|}{ Smokers $(N=99)$} \\
\hline $\begin{array}{l}\text { SNP } \\
\text { genotype }\end{array}$ & $\begin{array}{l}\text { Wild type } \\
(N=111)\end{array}$ & $\begin{array}{l}\text { Mutation type } \\
(N=169)\end{array}$ & AOR (95\% CI) & $\begin{array}{l}\text { Wild type } \\
(N=50)\end{array}$ & $\begin{array}{l}\text { Mutation type } \\
(N=131)\end{array}$ & AOR $(95 \%$ CI) & $\begin{array}{l}\text { Wild type } \\
(N=61)\end{array}$ & $\begin{array}{l}\text { Mutation } \\
\text { type }(N=38)\end{array}$ & AOR $(95 \% \mathrm{CI})$ \\
\hline \multicolumn{10}{|l|}{ rs1412125 } \\
\hline TT & $59(53.2 \%)$ & $89(52.7 \%)$ & 1.00 & $28(56.0 \%)$ & $67(51.1 \%)$ & 1.00 & $31(50.8 \%)$ & $22(57.9 \%)$ & 1.00 \\
\hline $\mathrm{TC}$ & $43(38.7 \%)$ & $66(39.1 \%)$ & $0.82(0.44 \sim 1.54)$ & $20(40.0 \%)$ & $53(40.5 \%)$ & $0.76(0.29 \sim 1.95)$ & $23(37.7 \%)$ & $13(34.2 \%)$ & $0.82(0.21 \sim 3.18)$ \\
\hline CC & $9(8.1 \%)$ & $14(8.2 \%)$ & $0.95(0.27 \sim 3.32)$ & $2(4.0 \%)$ & $11(8.4 \%)$ & $3.55(0.28 \sim 45.12)$ & $7(11.5 \%)$ & $3(7.9 \%)$ & $0.23(0.02 \sim 3.00)$ \\
\hline $\mathrm{TC}+\mathrm{CC}$ & $52(46.8 \%)$ & $80(47.3 \%)$ & $0.84(0.46 \sim 1.54)$ & $22(44.0 \%)$ & $64(48.9 \%)$ & $0.89(0.35 \sim 2.22)$ & $30(49.2 \%)$ & $16(42.1 \%)$ & $0.64(0.18 \sim 2.29)$ \\
\hline \multicolumn{10}{|l|}{ rs2249825 } \\
\hline $\mathrm{CC}$ & $74(66.7 \%)$ & $127(75.1 \%)$ & 1.00 & $39(78.0 \%)$ & $95(72.5 \%)$ & 1.00 & $35(57.4 \%)$ & $32(84.2 \%)$ & 1.00 \\
\hline CG & $34(30.6 \%)$ & $37(21.9 \%)$ & $0.55(0.27 \sim 1.10)$ & $10(20.0 \%)$ & $33(25.2 \%)$ & $1.19(0.41 \sim 3.50)$ & $24(39.3 \%)$ & $4(10.5 \%)$ & $0.07(0.01 \sim 0.74)^{*}$ \\
\hline GG & $3(2.7 \%)$ & $5(3.0 \%)$ & $2.06(0.36 \sim 11.87)$ & $1(2.0 \%)$ & $3(2.3 \%)$ & $1.52(0.09 \sim 27.00)$ & $2(3.3 \%)$ & $2(5.3 \%)$ & $2.21(0.09 \sim 57.15)$ \\
\hline $\mathrm{CG}+\mathrm{GG}$ & $37(33.3 \%)$ & $42(24.9 \%)$ & $0.63(0.32 \sim 1.23)$ & $11(22.0 \%)$ & $36(27.5 \%)$ & $1.22(0.43 \sim 3.45)$ & $26(42.6 \%)$ & $6(15.8 \%)$ & $0.16(0.03 \sim 1.02)$ \\
\hline \multicolumn{10}{|l|}{ rs1045411 } \\
\hline $\mathrm{CC}$ & $62(55.9 \%)$ & $103(60.9 \%)$ & 1.00 & $33(66.0 \%)$ & $76(58.0 \%)$ & 1.00 & $29(47.5 \%)$ & $27(71.1 \%)$ & 1.00 \\
\hline $\mathrm{CT}$ & $41(36.9 \%)$ & $56(33.1 \%)$ & $0.80(0.42 \sim 1.52)$ & $14(28.0 \%)$ & $48(36.6 \%)$ & $2.23(0.84 \sim 5.92)$ & $27(44.3 \%)$ & $8(21.1 \%)$ & $0.12(0.02 \sim 0.69)^{*}$ \\
\hline TT & $8(7.2 \%)$ & $10(6.0 \%)$ & $1.12(0.33 \sim 3.81)$ & $3(6.0 \%)$ & $7(5.4 \%)$ & $0.76(0.13 \sim 4.48)$ & $5(8.2 \%)$ & $3(7.9 \%)$ & $0.71(0.06-8.89)$ \\
\hline $\mathrm{CT}+\mathrm{TT}$ & $49(44.1 \%)$ & $66(39.1 \%)$ & $0.84(0.46 \sim 1.55)$ & $17(34.0 \%)$ & $55(42.0 \%)$ & $1.83(0.75 \sim 4.47)$ & $32(52.5 \%)$ & $11(28.9 \%)$ & $0.38(0.15 \sim 0.96)^{*}$ \\
\hline \multicolumn{10}{|l|}{ rs1360485 } \\
\hline $\mathrm{TT}$ & $60(54.1 \%)$ & $94(55.6 \%)$ & 1.00 & $32(64.0 \%)$ & $69(52.7 \%)$ & 1.00 & $28(45.9 \%)$ & $25(65.8 \%)$ & 1.00 \\
\hline $\mathrm{TC}$ & $40(36.0 \%)$ & $64(37.9 \%)$ & $1.05(0.55 \sim 1.97)$ & $15(30.0 \%)$ & $55(42.0 \%)$ & $3.07\left(1.12 \sim 8.38^{*}\right)$ & $25(41.0 \%)$ & $9(23.7 \%)$ & $0.12(0.02 \sim 0.72)^{*}$ \\
\hline $\mathrm{CC}$ & $11(9.9 \%)$ & $11(6.5 \%)$ & $0.96(0.29 \sim 3.16)$ & $3(6.0 \%)$ & $7(5.3 \%)$ & $0.89(0.15 \sim 5.34)$ & $8(13.1 \%)$ & $4(10.5 \%)$ & $0.51(0.05 \sim 5.11)$ \\
\hline $\mathrm{TC}+\mathrm{CC}$ & $51(45.9 \%)$ & $75(44.4 \%)$ & $1.03(0.56 \sim 1.89)$ & $18(36.0 \%)$ & $62(47.3 \%)$ & $2.47(0.98 \sim 6.20)$ & $33(54.1 \%)$ & $13(34.2 \%)$ & $0.18(0.04 \sim 0.86)^{*}$ \\
\hline
\end{tabular}

The adjusted odds ratios (AORs) with $95 \%$ confidence intervals (CIs) were estimated by multiple logistic regression models after controlling for age and gender.

Note: Bold text indicates a significant association with $p$ value of $<0.05$.

Abbreviations: SNP, single-nucleotide polymorphism.

\section{Associations between HMGBI genotypes and EGFR mutations in adenocarcinoma patients who did or did not smoke}

Regardless of sex, cigarette smoking is a major risk factor for lung cancer, and cigarette smoking was reported to affect the EGFR status in lung adenocarcinoma patients [24]. In this study, we divided our recruited lung adenocarcinoma patients into smoking and non-smoking groups and further investigated the difference between HMGB1 polymorphisms and EGFR status in these two groups. Distributions of HMGB1 genetic polymorphisms in the promoter region (rs1412125, -1615T/C), intron 1 (rs2249825, 3814C/G), and the 3' UTR (rs1045411, 2262C/T and rs1360485, T/C) in patients with the WT or mutant EGFR are shown in Table 2. Alleles with the highest distribution frequency of rs1412125, rs2249825, rs1045411, and rs1360485 in our recruited adenocarcinoma patients with the WT or mutant EGFR were homozygous for TT, CC, CC, and TT, respectively. For rs1412125 polymorphisms, there was no significant association demonstrated between variant types $(\mathrm{TC}, \mathrm{CC}$, and $\mathrm{TC}+\mathrm{CC})$ and the EGFR mutation type. In the smoking group, subjects with the HMGB1 polymorphic rs2249825 CG, rs1045411 CT, and rs1360485 TC genotypes exhibited significantly $(p<0.05)$ lower frequencies of 0.07 - $(95 \%$ CI: 0.01 0.74), 0.12- (95\% CI: 0.02 0.69), and 0.12-fold (95\% CI: 0.02 0.72) respectively, of having an EGFR mutation compared to their corresponding WT homozygotes (Table 2). In the non-smoking and overall cohorts we recruited, we observed that there was no significant difference in having an EGFR mutation in individuals with rs2249825 and rs1045411 polymorphisms of the HMGB1 gene compared to WT individuals (Table 2).

\section{Associations between HMGBI SNPs and EGFR hotspot mutations among lung adenocarcinoma patients}

Two most common EGFR hotspot mutations, L858R and an in-frame deletion, were also observed in most $(95 \%)$ of our recruited patients with EGFR mutations. We further assessed associations of the L858R mutation or in-frame deletion of the EGFR gene with HMGB1 genotypes. The CG and CG+GG genotypes of HMGB1 rs2249825 were significantly associated with a decreased proportion of in-frame deletions (adjusted odds ratio (AOR) $=0.473,95 \%$ CI: $0.228 \sim 0.979, \quad p=0.044 ; \quad$ AOR $=0.463, \quad 95 \% \quad$ CI: $0.227 \sim 0.941, p=0.033)$, but not with the L858R mutation (Table 3). In contrast, no significant association was observed of HMGB1 rs1412125, rs1045411, or rs1360485 polymorphisms with either hotspot mutations of the EGFR (Table 3).

\section{Correlations between polymorphic genotypes of $H M G B I$ and the clinical status of lung adenocarcinoma patients with EGFR mutations}

Impacts of the variant HMGB1 genotypes on the clinicopathological development of NSCLC harboring EGFR mutations, such as clinical cancer stage, primary tumor size, lymph node, distant metastasis, 
and degree of tumor cell differentiation, were further evaluated, and results are shown in Table 4. We classified patients into two subgroups of patients who had the homozygous WT alleles or who had at least one polymorphic allele. Among 169 lung adenocarcinoma patients with an EGFR mutation who carried at least one polymorphic C allele of HMGB1 rs1360485, they showed a significantly higher risk of having a poorly differentiated carcinoma (5.493-fold, 95\% CI: 1.130 26.696, $p=0.019)$ than those carrying the WT gene (Table 4). Next, we analyzed the synergistic effects of cigarette smoking combined with rs1360485 SNPs in lung adenocarcinoma patients with an EGFR mutation. Sixty patients who smoked cigarettes and harbored at least one polymorphic C allele of HMGB1 rs1360485 had an enhanced risk (9.9-fold, 95\% CI: 1.109 88.339, $p=0.016$ ) for developing poorly differentiated tumors compared to patients with TT homozygotes (Table 4). However, no significant associations between rs1360485 gene polymorphisms and the clinicopathologic status were observed in 109 non-smoking patients with EGFR mutations.

Table 3. Associations between the polymorphisms of high-mobility group box $1(H M G B I)$ and the epidermal growth factor receptor hotspot mutations in lung adenocarcinoma patients.

\begin{tabular}{|c|c|c|c|c|c|}
\hline \multirow[t]{2}{*}{ Variable } & \multirow{2}{*}{$\begin{array}{l}\text { Wild type } \\
(N=111)(\%)\end{array}$} & \multirow{2}{*}{$\begin{array}{l}\text { L858R } \\
(N=79)(\%)\end{array}$} & \multirow[b]{2}{*}{ AOR $(95 \%$ CI) } & \multicolumn{2}{|c|}{ Exon 19 in-frame deletion } \\
\hline & & & & $(N=81)(\%)$ & AOR $(95 \% \mathrm{CI})$ \\
\hline \multicolumn{6}{|l|}{ rs1412125 } \\
\hline TT & $59(53.2 \%)$ & $39(49.4 \%)$ & 1.00 & $46(56.8 \%)$ & 1.00 \\
\hline $\mathrm{TC}$ & $43(38.7 \%)$ & $34(43.0 \%)$ & $1.033(0.522 \sim 2.044)$ & $28(34.6 \%)$ & $0.640(0.328 \sim 1.250)$ \\
\hline $\mathrm{CC}$ & $9(8.1 \%)$ & $6(7.6 \%)$ & $1.109(0.319 \sim 3.852)$ & $7(8.6 \%)$ & $0.870(0.290 \sim 2.608)$ \\
\hline $\mathrm{TC}+\mathrm{CC}$ & $52(46.8 \%)$ & $40(50.6 \%)$ & $1.045(0.545 \sim 2.003)$ & $35(43.2 \%)$ & $0.683(0.366 \sim 1.272)$ \\
\hline \multicolumn{6}{|l|}{ rs2249825 } \\
\hline $\mathrm{CC}$ & $74(66.7 \%)$ & $55(69.6 \%)$ & 1.00 & $65(80.2 \%)$ & 1.00 \\
\hline CG & $34(30.6 \%)$ & $21(26.6 \%)$ & $0.767(0.370 \sim 1.591)$ & $15(18.5 \%)$ & $0.473(0.228 \sim 0.979)^{*}$, a \\
\hline GG & $3(2.7 \%)$ & $3(3.8 \%)$ & $1.234(0.196 \sim 7.757)$ & $1(1.2 \%)$ & $0.349(0.032 \sim 3.811)$ \\
\hline $\mathrm{CG}+\mathrm{GG}$ & $37(33.3 \%)$ & $24(30.4 \%)$ & $0.804(0.397 \sim 1.626)$ & $16(19.8 \%)$ & $0.463(0.227 \sim 0.941)^{*}, \mathrm{~b}$ \\
\hline \multicolumn{6}{|l|}{ rs1045411 } \\
\hline $\mathrm{CC}$ & $62(55.9 \%)$ & $44(55.7 \%)$ & 1.00 & $52(64.2 \%)$ & 1.00 \\
\hline $\mathrm{CT}$ & $41(36.9 \%)$ & $29(36.7 \%)$ & $0.913(0.457 \sim 1.823)$ & $26(32.1 \%)$ & $0.766(0.402 \sim 1.459)$ \\
\hline TT & $8(7.2 \%)$ & $6(7.6 \%)$ & $0.963(0.275 \sim 3.370)$ & $3(3.7 \%)$ & $0.448(0.106 \sim 1.884)$ \\
\hline $\mathrm{CT}+\mathrm{TT}$ & $49(44.1 \%)$ & $35(44.3 \%)$ & 0.921 (0.477 1.779) & $29(35.8 \%)$ & $0.715(0.385 \sim 1.330)$ \\
\hline \multicolumn{6}{|l|}{ rs1360485 } \\
\hline $\mathrm{TT}$ & $60(54.1 \%)$ & $42(53.2 \%)$ & 1.00 & $46(56.8 \%)$ & 1.00 \\
\hline $\mathrm{TC}$ & $40(36.0 \%)$ & $31(39.2 \%)$ & $0.989(0.493 \sim 1.986)$ & $31(38.3 \%)$ & $1.042(0.549 \sim 1.977)$ \\
\hline $\mathrm{CC}$ & $11(9.9 \%)$ & $6(7.6 \%)$ & $0.843(0.252 \sim 2.820)$ & $4(4.9 \%)$ & $0.498(0.140 \sim 1.769)$ \\
\hline $\mathrm{TC}+\mathrm{CC}$ & $51(45.9 \%)$ & $37(46.8 \%)$ & $0.961(0.496 \sim 1.864)$ & $35(43.2 \%)$ & $0.929(0.504 \sim 1.714)$ \\
\hline
\end{tabular}

The adjusted odds ratios (AORs) with $95 \%$ confidence intervals (CIs) were estimated by multiple logistic regression models after controlling for age and gender.

Note: Bold text indicates a significant association with a $p$ value of $<0.05$.

Abbreviations: SNP, single-nucleotide polymorphism.

a $p$ value $=0.044 ;{ }^{\mathrm{b}} p$ value $=0.033$.

Table 4. Associations between polymorphic genotypes of high-mobility group box 1 (HMGBI) (rs1360485) and clinicopathologic characteristics of lung cancer with epidermal growth factor receptor (EGFR) mutations.

\begin{tabular}{|c|c|c|c|c|c|c|c|c|c|}
\hline \multirow[t]{2}{*}{ Variable } & \multicolumn{3}{|c|}{ EGFR mutation $(N=169)$} & \multicolumn{3}{|c|}{ EGFR mutation with non-smoking $(N=109)$} & \multicolumn{3}{|c|}{ EGFR mutation with smoking $(N=60)$} \\
\hline & TT $(N=94)$ & $\mathrm{TC}+\mathrm{CC}(N=75)$ & $p$ value & TT $(N=60)$ & $\mathrm{TC}+\mathrm{CC}(N=49)$ & $p$ value & TT $(N=34)$ & $\mathrm{TC}+\mathrm{CC}(N=26)$ & $p$ value \\
\hline \multicolumn{10}{|l|}{ Stage } \\
\hline $\mathrm{I}+\mathrm{II}$ & $30(31.9 \%)$ & $17(22.7 \%)$ & $p=0.182$ & $20(33.3 \%)$ & $12(24.5 \%)$ & $p=0.313$ & $10(29.4 \%)$ & $5(19.2 \%)$ & $p=0.367$ \\
\hline $\mathrm{III}+\mathrm{IV}$ & $64(68.1 \%)$ & $58(77.3 \%)$ & & $40(66.7 \%)$ & $37(75.5 \%)$ & & $24(70.6 \%)$ & $21(80.8 \%)$ & \\
\hline \multicolumn{10}{|l|}{ Tumor T status } \\
\hline $\mathrm{T} 1+\mathrm{T} 2$ & $62(66.0 \%)$ & $46(61.3 \%)$ & $p=0.534$ & $40(66.7 \%)$ & $33(67.3 \%)$ & $p=0.940$ & $22(64.7 \%)$ & $13(50.0 \%)$ & $p=0.252$ \\
\hline $\mathrm{T} 3+\mathrm{T} 4$ & $32(34.0 \%)$ & $29(38.7 \%)$ & & $20(33.3 \%)$ & $16(32.7 \%)$ & & $12(35.3 \%)$ & $13(50.0 \%)$ & \\
\hline \multicolumn{10}{|l|}{ Lymph node status } \\
\hline Negative & $34(36.2 \%)$ & $20(26.7 \%)$ & $p=0.188$ & $24(40.0 \%)$ & $14(28.6 \%)$ & $p=0.213$ & $10(29.4 \%)$ & $6(23.1 \%)$ & $p=0.582$ \\
\hline Positive & $60(63.8 \%)$ & $55(73.3 \%)$ & & $36(60.0 \%)$ & $35(71.4 \%)$ & & $24(70.6 \%)$ & $20(76.9 \%)$ & \\
\hline \multicolumn{10}{|l|}{ Distant metastasis } \\
\hline Negative & $47(50.0 \%)$ & $33(44.0 \%)$ & $p=0.438$ & $32(53.3 \%)$ & $22(44.9 \%)$ & $p=0.381$ & $15(44.1 \%)$ & $11(42.3 \%)$ & $p=0.889$ \\
\hline Positive & $47(50.0 \%)$ & $42(56.0 \%)$ & & $28(46.7 \%)$ & $27(55.1 \%)$ & & $19(55.9 \%)$ & $15(57.7 \%)$ & \\
\hline \multicolumn{10}{|l|}{ Cell differentiation } \\
\hline Well + Moderately & $92(97.9 \%)$ & $67(89.3 \%)$ & $p=0.019^{*, \mathrm{a}}$ & $59(98.3 \%)$ & $47(95.9 \%)$ & $p=0.443$ & $33(97.1 \%)$ & $20(76.9 \%)$ & $p=0.016^{*, \mathrm{~b}}$ \\
\hline Poorly & $2(2.1 \%)$ & $8(10.7 \%)$ & & $1(1.7 \%)$ & $2(4.1 \%)$ & & $1(2.9 \%)$ & $6(23.1 \%)$ & \\
\hline
\end{tabular}

* $p$ value of $<0.05$ as statistically significant.

a Odds ratio (OR) (95\% confidence interval (CI)) : 5.493 (1.130 26.696); b OR (95\% CI): 9.900 (1.109 88.339). 


\section{Discussion}

Increasing evidence has shown that HMGB1 plays a key role in various cancer types including NSCLC [18], and HMGB1 dysfunction affects tumor development and therapy through regulating several hallmarks of cancer, such as metastasis, angiogenesis, resisting cell death, genome instability, mutations, and so on [15]. HMGB1 was characterized as having both oncogenic and tumor-suppressive roles in promoting both cell survival and death by regulating multiple signaling pathways, including genome stability, inflammation, immunity, proliferation, apoptosis, autophagy, metastasis, and metabolism $[15,17]$. Moreover, the HMGB1 expression level was reported to be inversely correlated with tumor differentiation [25]. In the past few years, several studies evaluated the association of HMGB1 polymorphisms with cancer susceptibility. In total, four frequently used SNPs within the human HMGB1 gene, rs1412125, rs2249825, rs1045411, and rs1360485, were studied for their associations with various cancer types including oral [26], liver [27], cervical [28], colorectal [29], and lung [30] cancers, especially in in Chinese Han population [20]. These four HMGB1 SNPs with minor allele frequencies were of $0.05(5 \%)$ or greater in the Chinese Han Beijing population. Moreover, previous GWAS study indicated that an association might exist between rs1360485 and rs1412125 SNPs with lung cancer risk [31] and rs1360485 showed a strong linkage disequilibrium with rs1045411 and rs2249825 [30, 32]. In lung cancer, knockdown of HMGB1 can increase the chemosensitivity of cells [33]. Moreover, the rs1412125 and rs2249825 polymorphisms were recently reported to be associated with the response to platinum-based chemotherapy in Chinese population [34]. High frequency of EGFR mutations in NSCLC was found in Asian population and the EGFR mutation of NSCLC was demonstrated to be a predictor of responsiveness to targeted therapy. Overexpression of HMGB1 was reported to promote cell proliferation and invasion in EGFR-mutant lung adenocarcinoma cells [35]. Based on the above annotations, we first investigated the association of EGFR mutation statuses in lung adenocarcinoma with variations (rs1412125, rs2249825, rs1045411, and rs1360485) of HMGB1 and their interactions with subjects who smoke in a Taiwanese population. In this study, our recruited cohort revealed a high prevalence of EGFR mutations in females and subjects who had never smoked and who harbored a more-differentiated tumor type. Characteristics of our recruited cohort were similar to Asian NSCLC patients as previously reported [36].
As to correlations between HMGB1 SNPs and frequencies of EGFR mutations, variant types (CG or $\mathrm{CG}+\mathrm{GG})$ of HMGB1 3814C/G polymorphism (rs2249825) were significantly negatively correlated with the EGFR mutation type of an exon 19 in-frame deletion. Furthermore, we observed that the rs2249825 C/G, rs1045411 C/T, and rs1045411 T/C heterozygous polymorphisms were associated with a significantly lower proportion of developing EGFR mutations compared to their respective homozygous $\mathrm{C} / \mathrm{C}, \mathrm{C} / \mathrm{C}$, and $\mathrm{T} / \mathrm{T}$ polymorphisms in the smoking population. These results suggested that the respective $\mathrm{G}, \mathrm{T}$, and $\mathrm{C}$ alleles of rs2249825, rs1045411, and rs1360485 might be protective factors against developing EGFR mutations in individuals who smoke. Moreover, individuals who smoke and carry at least one C allele of rs1360485 had a higher risk of having a poorly differentiated tumor type. Cigarette smoking, the best-known causative factor of lung cancer, was reported to induce HMGB1 upregulation in the blood and lung tissues of smokers [37]. A carcinogen of cigarette smoke, benzo[a]pyrene, can cause DNA damage, and base excision repair is a critical repair pathway that removes benzo[a]pyrene-induced DNA damages [38]. It was postulated that impaired base excision repair may be associated with increased genomic instability and increased tumor mutation rates such as EGFR mutations, and HMGB1 was reported to play an important role in base excision repair [39]. Based on these findings, we suggest that HMGB1 SNPs might affect cigarette smoke-induced HMGB1 expression to further decrease genomic instability and tumor EGFR mutations. Actually, expression of another base excision repair gene, excision repair cross complementing 1, was reported to be inversely correlated with EGFR mutations in NSCLC patients [40].

Previous studies demonstrated that polymorphisms of certain genes can influence gene expressions and biological functions. The C-to-G variation of the rs2249825 polymorphism, located in intron 1, might affect the binding site of v-myb and provide transcriptional control to enhance HMGB1 expression [34]. The rs2249825 polymorphism was also correlated with lipopolysaccharide-induced HMGB1 production in neutrophils [41]. In addition to rs2249825, two closely related SNPs, rs1045411 and rs1360485, showed strong linkage, are located in the 3' UTR of the HMGB1 gene, and may alter HMGB1 messenger (m)RNA expression through micro (mi)RNA binding. For example, rs1045411 is in close proximity to the mir-505 binding site, and a significant influence of $\mathrm{CT} / \mathrm{CC}$ genotypes of rs1045411 was reported to alter HMGB1 gene 
expression by regulating miR-505-binding activity [32]. Until now, the rs2249825 C/G polymorphism was reported to enhance HMGB1 expression, and the functional role of this SNP might have prevented EGFR mutations in our study cohort by maintaining genomic stability. In smokers with EGFR-mutant adenocarcinoma, not only rs2249825, but also rs1045411 and rs1360485 SNPs were all correlated with lower incidences of EGFR mutations. Although the rs1045411 SNP was reported to decrease HMGB1 expression by miR-505 [32], cigarette smoking might influence other miRNAs $[42,43]$ to regulate HMGB1 expression. Moreover, rs1360485 polymorphisms, TC and CC genotypes, were reported to reduce risk of lung adenocarcinoma, but there was no addictive interaction between rs1360485 polymorphisms and tobacco smoking [31]. Until now, interactions among tobacco consumption, rs1045411 and rs1360485 polymorphisms, and HMGB1 expression are unclear and worthy of future investigations.

In conclusion, in a Taiwanese population, we first identified associations among HMGB1 genetic polymorphisms, cigarette smoking, and the EGFR mutation status. Our results indicated that the exon 19 in-frame deletion mutation of the EGFR was associated with polymorphisms of the HMGB1 gene related to carcinogen detoxification and DNA repair in lung adenocarcinoma patients who smoked. These findings provide novel insights into the genesis of EGFR mutations and can be utilized as a predictor of responsiveness to EGFR targeted therapy.

\section{Competing Interests}

The authors have declared that no competing interest exists.

\section{References}

[1] Hoffman RM, Sanchez R. Lung Cancer Screening. Med Clin North Am 2017; 101: 769-785.

[2] Wood SL, Pernemalm M, Crosbie PA, Whetton AD. Molecular histology of lung cancer: from targets to treatments. Cancer Treat Rev 2015; 41: 361-375.

[3] Furrukh M. Tobacco Smoking and Lung Cancer: Perception-changing facts. Sultan Qaboos Univ Med J 2013; 13: 345-358.

[4] Fang X, Yin Z, Li X, Xia L, Quan X, Zhao Y, et al. Multiple functional SNPs in differentially expressed genes modify risk and survival of non-small cell lung cancer in Chinese female non-smokers. Oncotarget 2017; 8: 18924-18934.

[5] Alberg AJ, Brock MV, Ford JG, Samet JM, Spivack SD. Epidemiology of lung cancer: Diagnosis and management of lung cancer, 3rd ed: American College of Chest Physicians evidence-based clinical practice guidelines. Chest 2013; 143: e1S-e29S.

[6] Wee P, Wang Z. Epidermal Growth Factor Receptor Cell Proliferation Signaling Pathways. Cancers (Basel) 2017; 9:

[7] Siegelin MD, Borczuk AC. Epidermal growth factor receptor mutations in lung adenocarcinoma. Lab Invest 2014; 94: 129-137.

[8] Bashir NA, Ragab ES, Khabour OF, Khassawneh BY, Alfaqih MA, Momani JA. The Association between Epidermal Growth Factor Receptor (EGFR) Gene Polymorphisms and Lung Cancer Risk. Biomolecules 2018; 8:

[9] Shigematsu H, Lin L, Takahashi T, Nomura M, Suzuki M, Wistuba II, et al. Clinical and biological features associated with epidermal growth factor receptor gene mutations in lung cancers. J Natl Cancer Inst 2005; 97: 339-346.

[10] Isobe K, Hata Y, Tochigi N, Kaburaki K, Kobayashi H, Makino T, et al. Clinical significance of BIM deletion polymorphism in non-small-cell lung cancer with epidermal growth factor receptor mutation. J Thorac Oncol 2014; 9: 483-487.
[11] Yang SY, Yang TY, Li YJ, Chen KC, Liao KM, Hsu KH, et al. EGFR exon 19 in-frame deletion and polymorphisms of DNA repair genes in never-smoking female lung adenocarcinoma patients. Int J Cancer 2013; 132: 449-458.

[12] Huang CY, Hsieh MJ, Wu WJ, Chiang WL, Liu TC, Yang SF, et al. Association of endothelial nitric oxide synthase (eNOS) polymorphisms with EGFR-mutated lung adenocarcinoma in Taiwan. J Cancer 2018; 9: 2518-2524.

[13] Chatterjee N, Walker GC. Mechanisms of DNA damage, repair, and mutagenesis. Environ Mol Mutagen 2017; 58: 235-263.

[14] Lanuszewska J, Widlak P. High mobility group 1 and 2 proteins bind preferentially to DNA that contains bulky adducts induced by benzo[a]pyrene diol epoxide and N-acetoxy-acetylaminofluorene. Cancer Lett 2000; 158: 17-25.

[15] Kang R, Zhang Q, Zeh HJ, 3rd, Lotze MT, Tang D. HMGB1 in cancer: good, bad, or both? Clin Cancer Res 2013; 19: 4046-4057.

[16] Lange SS, Mitchell DL, Vasquez KM. High mobility group protein B1 enhances DNA repair and chromatin modification after DNA damage. Proc Natl Acad Sci U S A 2008; 105: 10320-10325.

[17] He SJ, Cheng J, Feng X, Yu Y, Tian L, Huang Q. The dual role and therapeutic potential of high-mobility group box 1 in cancer. Oncotarget 2017; 8: 64534-64550.

[18] Feng A, Tu Z, Yin B. The effect of HMGB1 on the clinicopathological and prognostic features of non-small cell lung cancer. Oncotarget 2016; 7: 20507-20519.

[19] Wu L, Yang L. The function and mechanism of HMGB1 in lung cancer and its potential therapeutic implications. Oncol Lett 2018; 15: 6799-6805.

[20] Kumari T, Kumar B. High-mobility group box 1 protein (HMGB1) gene polymorphisms and cancer susceptibility: A comprehensive meta-analysis. Clin Chim Acta 2018; 483: 170-182.

[21] Su SC, Hsieh MJ, Lin CW, Chuang CY, Liu YF, Yeh CM, et al. Impact of HOTAIR Gene Polymorphism and Environmental Risk on Oral Cancer. J Dent Res 2018; 97: 717-724.

[22] Su CW, Chien MH, Lin CW, Chen MK, Chow JM, Chuang CY, et al. Associations of genetic variations of the endothelial nitric oxide synthase gene and environmental carcinogens with oral cancer susceptibility and development. Nitric Oxide 2018; 79: 1-7.

[23] Hua KT, Liu YF, Hsu CL, Cheng TY, Yang CY, Chang JS, et al. 3'UTR polymorphisms of carbonic anhydrase IX determine the miR-34a targeting efficiency and prognosis of hepatocellular carcinoma. Sci Rep 2017; 7: 4466.

[24] Tseng CH, Chiang CJ, Tseng JS, Yang TY, Hsu KH, Chen KC, et al. EGFR mutation, smoking, and gender in advanced lung adenocarcinoma. Oncotarget 2017; 8: 98384-98393.

[25] Ando K, Sakoda M, Ueno S, Hiwatashi K, Iino S, Minami K, et al. Clinical Implication of the Relationship Between High Mobility Group Box-1 and Tumor Differentiation in Hepatocellular Carcinoma. Anticancer Res 2018; 38: 3411-3418.

[26] Supic G, Kozomara R, Zeljic K, Stanimirovic D, Magic M, Surbatovic M, et al. HMGB1 genetic polymorphisms in oral squamous cell carcinoma and oral lichen planus patients. Oral Dis 2015; 21: 536-543.

[27] Wang B, Yeh CB, Lein MY, Su CM, Yang SF, Liu YF, et al. Effects of HMGB1 Polymorphisms on the Susceptibility and Progression of Hepatocellular Carcinoma. Int J Med Sci 2016; 13: 304-309.

[28] Wu HH, Liu YF, Yang SF, Lin WL, Chen SC, Han CP, et al. Association of single-nucleotide polymorphisms of high-mobility group box 1 with susceptibility and clinicopathological characteristics of uterine cervical neoplasia in Taiwanese women. Tumour Biol 2016;

[29] Wang JX, Yu HL, Bei SS, Cui ZH, Li ZW, Liu ZJ, et al. Association of HMGB1 Gene Polymorphisms with Risk of Colorectal Cancer in a Chinese Population. Med Sci Monit 2016; 22: 3419-3425.

[30] Hu W, Liu PY, Yang YC, Chen PC, Su CM, Chao CC, et al. Association of HMGB1 Gene Polymorphisms with Lung Cancer Susceptibility and Clinical Aspects. Int J Med Sci 2017; 14: 1197-1202.

[31] Jiang M, Li X, Quan X, Li X, Zhou B. Single Nucleotide Polymorphisms in HMGB1 Correlate with Lung Cancer Risk in the Northeast Chinese Han Population. Molecules 2018; 23:

[32] Lin CW, Chou YE, Yeh CM, Yang SF, Chuang CY, Liu YF. A functional variant at the miRNA binding site in HMGB1 gene is associated with risk of oral squamous cell carcinoma. Oncotarget 2017; 8: 34630-34642.

[33] Zhang R, Li Y, Wang Z, Chen L, Dong X, Nie X. Interference with HMGB1 increases the sensitivity to chemotherapy drugs by inhibiting HMGB1-mediated cell autophagy and inducing cell apoptosis. Tumour Biol 2015; 36: 8585-8592.

[34] Wang Y, Li XP, Yin JY, Zhang Y, He H, Qian CY, et al. Association of HMGB1 and HMGB2 genetic polymorphisms with lung cancer chemotherapy response. Clin Exp Pharmacol Physiol 2014; 41: 408-415.

[35] Sun KK, Ji C, Li X, Zhang L, Deng J, Zhong N, et al. Overexpression of high mobility group protein $\mathrm{B} 1$ correlates with the proliferation and metastasis of lung adenocarcinoma cells. Mol Med Rep 2013; 7: 1678-1682.

[36] Kawaguchi T, Koh Y, Ando M, Ito N, Takeo S, Adachi H, et al. Prospective Analysis of Oncogenic Driver Mutations and Environmental Factors: Japan Molecular Epidemiology for Lung Cancer Study. J Clin Oncol 2016; 34: 2247-2257.

[37] Gangemi S, Casciaro M, Trapani G, Quartuccio S, Navarra M, Pioggia G, et al. Association between HMGB1 and COPD: A Systematic Review. Mediators Inflamm 2015; 2015: 164913. 
[38] Li W, Hu J, Adebali O, Adar S, Yang Y, Chiou YY, et al. Human genome-wide repair map of DNA damage caused by the cigarette smoke carcinogen benzo[a]pyrene. Proc Natl Acad Sci U S A 2017; 114: 6752-6757.

[39] Liu Y, Prasad R, Wilson SH. HMGB1: roles in base excision repair and related function. Biochim Biophys Acta 2010; 1799: 119-130.

[40] Gandara DR, Grimminger P, Mack PC, Lara PN, Jr., Li T, Danenberg PV, et al. Association of epidermal growth factor receptor activating mutations with low ERCC1 gene expression in non-small cell lung cancer. J Thorac Oncol 2010; 5: 1933-1938.

[41] Zeng L, Zhang AQ, Gu W, Chen KH, Jiang DP, Zhang LY, et al. Clinical relevance of single nucleotide polymorphisms of the high mobility group box 1 protein gene in patients with major trauma in southwest China. Surgery 2012; 151: 427-436.

[42] Schembri F, Sridhar S, Perdomo C, Gustafson AM, Zhang X, Ergun A, et al. MicroRNAs as modulators of smoking-induced gene expression changes in human airway epithelium. Proc Natl Acad Sci U S A 2009; 106: 2319-2324.

[43] Fujii T, Shimada K, Nakai T, Ohbayashi C. MicroRNAs in Smoking-Related Carcinogenesis: Biomarkers, Functions, and Therapy. J Clin Med 2018; 7: 